\title{
Problemas de salud mental en estudiantes de una universidad regional chilena
}

\author{
ZAYRA ANTÚNEZ ${ }^{1, \mathrm{a}}$, EUGENIA V. VINET ${ }^{2, \mathrm{~b}}$
}

\section{Mental health problems among students of a regional Chilean university}

Background: The increase in university population in Chile has also increased the number of students presenting mental health disorders. Aim: To determine the frequency of mental health problems and its association with other relevant variables among students of a regional university in Chile. Material and Methods: A sample of 484 students aged 18 to 28 years (45\% males) were assessed once using a battery of tests designed to detect mental health problems. The instruments used were Derogates SCL-90-R, Beck Anxiety Inventory (BAI), Beck Depression Inventory (BDI-II), Depression, Anxiety and Stress Scales (DASS-21) and a social-demographic questionnaire. Results: Fifteen percent of the sample presented high levels of mental health symptoms, determined according to the cut-off points of Beck inventories. This symptomatic group, which also showed high levels of stress, was composed mostly of freshmen and women. There was a significant association between symptoms and socio-demographic variables such as living in rural areas, ethnicity and socioeconomic status, a higher need for psychological and psychiatric care and a lower number of extracurricular activities. Conclusions: The knowledge of mental health problems among university students will allow to generate strategies for mental health prevention, promotion and treatment.

(Rev Med Chile 2013; 141: 209-216).

Key words: Demography, health status; Diagnosis, dual (Psychiatry); Education, professional; Mental health.

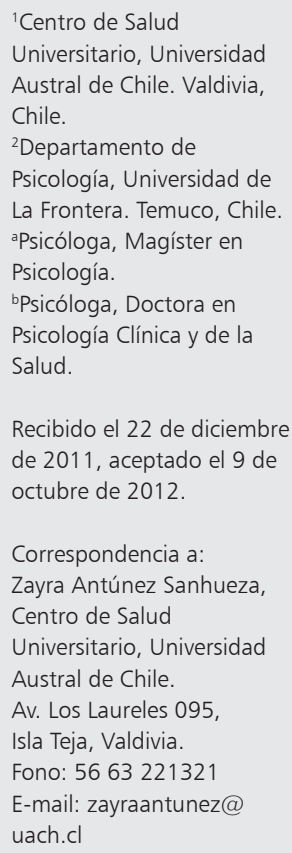

Recibido el 22 de diciembre de 2011, aceptado el 9 de octubre de 2012.

Correspondencia a: Zayra Antúnez Sanhueza,

Centro de Salud

Universitario, Universidad

Austral de Chile.

Av. Los Laureles 095,

Isla Teja, Valdivia.

Fono: 5663221321

E-mail: zayraantunez@

uach.cl

$\mathrm{E}$ 1 número de estudiantes universitarios se ha triplicado en Chile entre los años 1981 y $2000^{1}$. Posteriormente, la matrícula total de alumnos de pregrado ha aumentado de 435 mil estudiantes en el año 2000 a 768 mil en el año 2008 con un incremento de $76 \%$; de igual forma, la matrícula de estudiantes de primer año aumentó de 137 mil estudiantes a 249 mil en igual período, lo que equivale a un incremento de $82 \%{ }^{2}$. Esto implica también un mayor número de estudiantes que presentan algún trastorno de la salud mental; sin embargo confluyen aquí los siguientes factores adicionales.

La mayoría de los estudiantes universitarios de pregrado se encuentra en la etapa de la adolescencia tardía, período en el cual tienden a emerger con particular intensidad problemas de salud mental como los trastornos del estado de ánimo y los trastornos ansiosos ${ }^{3}$. Durante la adolescencia se acentúan las conductas de riesgo como el consumo abusivo, la dependencia de sustancias psicoactivas y las conductas "antisociales", se consolidan patrones desadaptativos como los trastornos de personalidad y existe mayor vulnerabilidad al desarrollo de trastornos alimentarios ${ }^{4}$. También algunas formas de psicosis, como la esquizofrenia, tienden a irrumpir en este período 5 . Además, entre la adolescencia y la adultez joven hay una mayor demanda de ajustes personales y sociales que aumentan el riesgo a desarrollar trastornos emocionales por estrés ${ }^{6}$. Particularmente en los universitarios, el riesgo de presentar cuadros 
psicopatológicos se relaciona con altos grados de exigencia, competitividad y expectativas que acrecientan el estrés, factor importante en la vida de estos jóvenes y que tiene un impacto negativo sobre su salud ${ }^{7-9}$.

Como existe consenso entre los profesionales que atienden las necesidades de salud de los estudiantes universitarios sobre su vulnerabilidad para desarrollar trastornos emocionales, a partir de 1990 se registran estudios empíricos que muestran las características generales de su salud mental y las alteraciones más frecuentes que ellos presentan. En un estudio realizado en la Universidad de La Frontera en $1992^{6}$ se encontró que 36,2\% de las mujeres y $28,9 \%$ de los hombres presentaron trastornos emocionales evaluados a través del Cuestionario de Salud de General ${ }^{10}$. Otro estudio, más reciente, de la Universidad de Concepción ${ }^{11}$, arrojó altas prevalencias para los trastornos depresivo y ansioso evaluados a través de los inventarios Beck $^{12,13}$; específicamente, $22 \%$ de las mujeres y $10,6 \%$ de los hombres presentó trastorno depresivo, en tanto que $23,8 \%$ de las mujeres y $10,7 \%$ de los hombres presentó trastorno ansioso. Otro estudio $^{14}$ de la década de 1990, encontró que 25\% de los estudiantes consumía alcohol en un nivel de riesgo; este dato es confirmado actualmente por el Consejo Nacional para el Control de Estupefacientes al señalar que los jóvenes universitarios chilenos son un grupo de riesgo con elevadas tasas de consumo de marihuana y alcohol ${ }^{15}$.

En estudios más circunscritos, se señala que 34,8\% de los estudiantes beneficiarios del Programa de Atención Económica (PAE) de la Universidad de Chile ${ }^{16}$, presentan signos inespecíficos de malestar emocional medido a través del Cuestionario de Salud General ${ }^{10}$. Por otro lado, entre los estudiantes que recibían atención psicológica y/o psiquiátrica en el Servicio de Salud Estudiantil de una universidad de Santiago, se encontró que $48,3 \%$ de ellos presentaba trastornos adaptativos, $22,8 \%$ trastornos del ánimo, 20,9\% trastornos de ansiedad y un $6,7 \%$ trastornos relacionados con sustancias, observándose frecuencias significativamente mayores en los hombres para trastornos de ansiedad y trastornos relacionados con sustancias ${ }^{17}$.

Considerando este contexto, el objetivo de esta investigación fue dimensionar la presencia de problemas de salud mental en la población general de estudiantes de una universidad regional perte- neciente al Consejo de Rectores; secundariamente se abordó la relación entre problemas de salud mental y un conjunto de variables sociodemográficas relevantes.

\section{Material y Método}

\section{Participantes}

La población correspondió a estudiantes de pregrado de la Universidad Austral de Chile (UACh), que cursaban entre primero y cuarto año de sus respectivas carreras. Se seleccionó una muestra representativa a través de un muestreo aleatorio por conglomerados, según carreras y niveles considerando un tamaño muestral con un nivel de confianza del 99\% $(z=2,576)$ para una población finita sin reposición. La muestra quedó constituida por 484 estudiantes y se trabajó con un diseño no experimental correlacional, de carácter transversal.

\section{Instrumentos}

Se utilizó un Cuestionario Sociodemográfico para recoger datos básicos: sexo, edad y nivel socioeconómico -Método de Evaluación del Nivel Socioeconómico ESOMAR ${ }^{18}$-, junto a otras variables de contexto para la salud mental, como tratamientos psicológicos o psiquiátricos previos, consumo de alcohol y otras drogas, participación en actividades recreativas o deportivas. Además los estudiantes respondieron los siguientes instrumentos:

1. Inventario de Sintomas de Derogatis, Revisado $(S C L-90-R)^{19}$. Es un autoinforme que evalúa el grado de malestar psicológico actual que experimenta una persona a través de 90 síntomas psiquiátricos de variado nivel de gravedad. El respondiente debe indicar en qué medida lo ha molestado o perturbado cada uno de esos problemas durante la última semana utilizando una escala tipo Likert de cinco puntos. Los resultados se organizan en nueve dimensiones primarias de síntomas (Somatización, Obsesiones, Sensitividad Interpersonal, Depresión, Ansiedad, Hostilidad, Ansiedad Fóbica, Ideación Paranoide y Psicoticismo) y tres índices globales de psicopatología: Índice de Gravedad Global (Global Severity Index, GSI), Índice de Malestar Positivo (Positive Symptom Distress, PSDI) y Total de Síntomas Positivos (Positive Symptom Total, PST). El SCL-90-R cuenta con una versión 
adaptada para población universitaria chilena con buenas características psicométricas y datos normativos propios ${ }^{20 .}$

2. Versión chilena abreviada de las Escalas de Depresión, Ansiedad y Estrés (DASS-21): Este instrumento (Versión Chilena Traducida y Adaptada por E. Vinet, L. Rehbein, M. Román y J. Saiz; Universidad de La Frontera, 2008) consta de 21 ítems, con cuatro alternativas de respuesta en formato Likert y fue validado recientemente en población universitaria chilena ${ }^{21}$. Su estructura y propiedades psicométricas han sido ampliamente analizadas en diversas investigaciones, presentando adecuadas propiedades psicométricas en países como $\mathrm{Ca}$ nadá $^{22,23}$, Portugal ${ }^{24}$, Estados Unidos ${ }^{25}$, España ${ }^{26}$.

3. Inventario de Ansiedad de Beck (BAI): Es un instrumento autoaplicable de 21 ítems, desarrollado para estimar ansiedad, discriminando entre ésta y la sintomatología depresiva ${ }^{13}$. Estudios realizados en Chile, en adolescentes ${ }^{27}$ como en universitarios ${ }^{11}$, han verificado sus propiedades psicométricas y su capacidad para discriminar efectivamente entre jóvenes con ansiedad normal y patológica, al utilizar un punto de corte de 16.

4. Inventario de Depresión de Beck, segunda edición (BDI-II): Este instrumento es ampliamente utilizado como medida de sintomatología depresiva en pacientes con trastornos psicológicos y en población no clínica desde los 13 años ${ }^{12}$. Consta de 21 ítems, es autoaplicable y presenta adecuadas propiedades psicométricas ${ }^{28,29}$. En Chile, ha demostrado discriminar efectivamente entre población clínica y no consultante con un puntaje de corte de 19, el cual indica presencia de sintomatología depresiva importante ${ }^{30}$.

\section{Procedimiento}

Se solicitó aprobación al Comité de Bioética de la Facultad de Medicina de la UACh. Posteriormente, se contactó a Directores de Escuela y docentes respectivos, para aplicar los instrumentos en período de clases. Los jóvenes convocados colaboraron voluntariamente en la investigación sin excepciones, formalizando su participación mediante la firma de un consentimiento informado. Las respuestas fueron confidenciales y anónimas, pero se les dio la opción de registrar datos personales para consultar sus resultados o ser contactados en caso de necesidad de evaluación y/o tratamiento médico o psicológico, el que podrían iniciar en el Centro de Salud Universitario de la UACh.

\section{Resultados}

Los datos fueron analizados mediante el programa SPSS, versión 11.5, utilizando análisis descriptivos y de comparación de grupos.

La muestra, constituida por 484 estudiantes, presentó las siguientes características sociodemográficas: Fueron jóvenes no consultantes entre 18 y 28 años (M: 20,2, DT: 2,1), con $44,8 \%$ de sexo masculino. Su procedencia fue principalmente urbana $(86,4 \%)$ con un porcentaje menor $(12,2 \%)$ de zonas rurales; además, un 14\% de ellos declaró pertenecer a la etnia mapuche. La mayoría de ellos era soltero (97\%) y su distribución socioeconómica correspondió principalmente al nivel medio ( $23 \%$ medio-bajo, $28 \%$ medio y $30 \%$ medio-alto). La distribución según año de ingreso mostró que la gran mayoría de los estudiantes cursaba primer año (46,4\%; ingreso 2009), con porcentajes decrecientes en segundo (20,5\%; ingreso 2008$)$, tercero (12,3\% , ingreso 2007$)$ y cuarto año $(9,4 \%$; ingreso 2006); además, 10,1\% había ingresado entre 2005 y 2001 y 1,2\% omitió su año de ingreso; estos estudiantes presentaban un retraso en el desarrollo regular de sus estudios.

En las variables que contextualizan la salud mental se observó que sólo 7,4\% de los estudiantes reportaba haber recibido tratamiento psiquiátrico y $20 \%$ tratamiento psicológico.

Respecto del consumo de alcohol y drogas (marihuana principalmente), en total, $75,5 \%$ de los estudiantes reportó consumir alcohol al menos una vez al mes, mientras que $15,9 \%$ reportó consumir algún tipo de droga en el mismo período. De ellos, 36,3\% consumiría alcohol y 2,9\% drogas entre 2 a 4 veces en el mes; además, $4,1 \%$ presentaría un consumo patológico de alcohol, es decir, dos o más veces por semana, mientras que 3,5\% consumiría drogas al menos dos veces por semana.

En contraste, en términos de autocuidado y recreación, el $60 \%$ de los jóvenes refirió no realizar ninguna actividad extraprogramática. El 32,7\% que realizaba al menos una actividad extraprogramática prefería las actividades deportivas o recreativas $(23,7 \%)$, las sociales $(10,3)$, culturales $(6,6 \%)$ y musicales $(4,3 \%)$.

El autoreporte de los tests aplicados entregó los resultados que se presentan en la Tabla 1 .

Estos resultados muestran que, en general, la población universitaria presentó escasa sintomatología significativa. Sin embargo, las mujeres 
Tabla 1. Estadísticos descriptivos generales y diferencias según sexo en las escalas del SCL-90-R, el DASS-21 y las escalas BAI y BDI-II

\begin{tabular}{|c|c|c|c|c|c|c|c|c|}
\hline \multirow[t]{2}{*}{ Escalas } & \multicolumn{2}{|c|}{$\begin{array}{l}\text { Muestra total } \\
\qquad n=484\end{array}$} & \multicolumn{2}{|c|}{$\begin{array}{c}\text { Hombres } \\
n=217\end{array}$} & \multicolumn{2}{|c|}{$\begin{array}{l}\text { Mujeres } \\
\mathbf{n}=\mathbf{2 6 7}\end{array}$} & \multirow{2}{*}{\multicolumn{2}{|c|}{$\begin{array}{l}\text { Diferencias según } \\
\text { género } \\
\text { t }\end{array}$}} \\
\hline & $\mathbf{M}$ & DE & $\mathbf{M}$ & DE & $\mathbf{M}$ & DE & & \\
\hline \multicolumn{9}{|l|}{ SCL-90-R } \\
\hline Somatización & 44,94 & 11,70 & 41,67 & 11,32 & 47,60 & 11,24 & $-5,75$ & $* * *$ \\
\hline Obsesiones & 50,60 & 16,21 & 48,65 & 13,64 & 52,16 & 17,94 & $-2,38$ & * \\
\hline Sens. Interper. & 48,59 & 16,55 & 46,40 & 11,51 & 50,40 & 19,60 & $-2,79$ & $* *$ \\
\hline Depresión & 47,17 & 14,69 & 43,85 & 11,81 & 49,84 & 16,22 & $-4,54$ & $* * *$ \\
\hline Ansiedad & 42,69 & 12,46 & 40,77 & 12,04 & 44,25 & 12,61 & $-3,08$ & $* *$ \\
\hline Hostilidad & 48,46 & 13,65 & 47,16 & 11,22 & 49,55 & 15,31 & $-1,92$ & \\
\hline Ans. Fóbica & 48,21 & 8,02 & 47,09 & 6,73 & 49,16 & 8,86 & $-2,94$ & $* *$ \\
\hline Ideac. Paran. & 47,90 & 9,45 & 47,68 & 9,43 & 48,08 & 9,48 & $-0,47$ & \\
\hline Psicoticismo & 45,87 & 9,84 & 45,59 & 10,38 & 46,04 & 9,24 & $-0,52$ & \\
\hline GSI & 44,36 & 13,36 & 41,96 & 13,17 & 46,30 & 13,20 & $-3,60$ & $* * *$ \\
\hline PSDI & 48,45 & 12,96 & 46,07 & 12,58 & 50,36 & 12,96 & $-3,66$ & $* * *$ \\
\hline PST & 43,61 & 12,33 & 41,86 & 12,80 & 45,05 & 11,77 & $-2,85$ & $* *$ \\
\hline \multicolumn{9}{|l|}{ DASS-21 } \\
\hline Depresión & 3,92 & 3,81 & 3,50 & 3,37 & 4,25 & 4,12 & $-2,18$ & $*$ \\
\hline Ansiedad & 3,13 & 3,13 & 2,67 & 2,66 & 3,48 & 3,38 & $-3,06$ & ** \\
\hline Estrés & 5,23 & 3,99 & 4,70 & 3,50 & 5,65 & 4,28 & $-2,77$ & $* *$ \\
\hline BAI & 9,82 & 7,44 & 8,29 & 6,31 & 11,06 & 8,03 & $-4,23$ & $* * *$ \\
\hline BDI-II & 14,96 & 9,53 & 13,06 & 8,79 & 16,49 & 9,88 & $-3,99$ & $* * *$ \\
\hline
\end{tabular}

Nota. Sens. Interper.: Sensitividad Interpersonal; Ans. Fóbica: Ansiedad Fóbica; Ideac. Paran.: Ideación Paranoide; GSI: Índice de Gravedad Global; PSDI: Índice de Malestar Positivo; PST: Total de Síntomas Positivos. ${ }^{*} p<0,05 ;{ }^{* *} p<0,01 ;{ }^{* * *} p<0,001$.

reportaron más sintomatología que los hombres y estas diferencias son muy significativas en las escalas que pesquisaban malestar general y sintomatología depresiva y ansiosa. Considerando este resultado se decidió realizar los análisis siguientes separadamente para cada sexo.

Dado el predominio de sintomatología ansiosa y depresiva se utilizaron los puntajes de corte de las pruebas de Beck para identificar los casos con mayor sintomatología. Tal como se presenta en la Tabla 2 , se encontró que $30,1 \%$ de la muestra total presentaría sintomatología depresiva y $20,9 \%$ sintomatología ansiosa, determinándose, además, que $15,5 \%$ de la muestra presentaría, conjuntamente, sintomatología depresiva y ansiosa y que estos trastornos se presentan predominantemente en estudiantes de primer año.

El grupo con sintomatología conjunta, formado por 25 hombres y 52 mujeres ( 3 omitieron año de ingreso), fue examinado detenidamente según las variables sociodemográficas y de contexto encontrándose algunas diferencias relevantes con respecto al resto de la población universitaria.

Este grupo, integrado mayoritariamente por estudiantes de primer año, presentó un incremento de la ruralidad ( $20 \%$ en los hombres) y de la pertenencia a la etnia mapuche (19,2\% en las mujeres) $y$, además, el nivel socioeconómico medio-bajo se vio incrementado ( $40 \%$ en hombres y $28,8 \%$ en mujeres) en desmedro de los niveles superiores. Un porcentaje importante de las mujeres reportó recibir atención psicológica y psiquiátrica $(34 \%$ y $17 \%$ respectivamente) y el número que no realizaba actividades extraprogramáticas fue mayor que en la población general universitaria (64\% en los hombres y $65,4 \%$ en las mujeres).

Este grupo, tal como muestra la Tabla 3, presentó incrementos muy significativos en la sintomalogía reportada a través de los instrumentos aplicados.

Aunque todas las puntuaciones obtenidas por este grupo indican sintomatología en salud mental, 
Salud mental en universitarios - Z. Antúnez et al

Tabla 2. Frecuencias y porcentajes de estudiantes de la muestra total que reportan sintomatología depresiva y/o ansiosa a través de las pruebas de Beck según año de ingreso a la universidad

\begin{tabular}{|c|c|c|c|c|c|c|}
\hline \multirow[t]{2}{*}{ Indicadores de sintomatología } & \multicolumn{2}{|c|}{$\begin{array}{c}\text { Muestra total } \\
n=478^{*}\end{array}$} & \multicolumn{2}{|c|}{$\begin{array}{l}\text { Hombres } \\
n=215^{*}\end{array}$} & \multicolumn{2}{|c|}{$\begin{array}{l}\text { Mujeres } \\
n=261^{*}\end{array}$} \\
\hline & $\mathbf{f}$ & $\%$ & $\mathbf{f}$ & $\%$ & $\mathbf{f}$ & $\%$ \\
\hline \multicolumn{7}{|l|}{ BDI-II sobre punto de corte 19} \\
\hline Ingreso 2009 & 72 & 50,0 & 20 & 38,5 & 52 & 56,5 \\
\hline Ingreso 2008 & 18 & 12,5 & 7 & 13,5 & 11 & 12,0 \\
\hline Ingreso 2007 & 19 & 13,2 & 9 & 17,3 & 10 & 10,8 \\
\hline Ingreso 2006 & 16 & 11,1 & 5 & 9,5 & 11 & 12,0 \\
\hline Ingreso en años anteriores & 19 & 13,2 & 11 & 21,2 & 8 & 8,7 \\
\hline Muestra total & 144 & 30,1 & 52 & 24,2 & 92 & 35.2 \\
\hline \multicolumn{7}{|l|}{ BAl sobre punto de corte 16} \\
\hline Ingreso 2009 & 56 & 56,0 & 20 & 60.6 & 36 & 54,5 \\
\hline Ingreso 2008 & 10 & 10,0 & 2 & 6,0 & 7 & 10,6 \\
\hline Ingreso 2007 & 18 & 18,0 & 5 & 15,2 & 13 & 19,7 \\
\hline Ingreso 2006 & 8 & 8,0 & 3 & 9,1 & 5 & 7,6 \\
\hline Ingreso en años anteriores & 8 & 8,0 & 3 & 9,1 & 5 & 7,6 \\
\hline Muestra total & 100 & 20.9 & 33 & 15.3 & 66 & 25.3 \\
\hline \multicolumn{7}{|l|}{ BDI y BAI sobre puntos de corte } \\
\hline Ingreso 2009 & 43 & 58,1 & 15 & 60,0 & 28 & 57,1 \\
\hline Ingreso 2008 & 6 & 8,1 & 2 & 8,0 & 4 & 8,2 \\
\hline Ingreso 2007 & 13 & 17,6 & 4 & 16,0 & 9 & 18,3 \\
\hline Ingreso 2006 & 6 & 8,1 & 2 & 8,0 & 4 & 8,2 \\
\hline Ingreso en años anteriores & 6 & 8,1 & 2 & 8,0 & 4 & 8,2 \\
\hline Muestra total & 74 & 15,5 & 25 & & 49 & 18,8 \\
\hline
\end{tabular}

*El $n$ de la Muestra total y de las muestras parciales de Hombres y Mujeres es menor que el de la Tabla 1 pues hay casos en los cuáles se omitió el año de ingreso.

son especialmente relevantes algunas diferencias observadas con respecto al resto de la población universitaria. En los hombres los incrementos mayores aparecen en las escalas de depresión e ideación paranoide del SCL-90-R $(t=12,25$ y 8,97 respectivamente) y estrés del DASS-21 $(t=9,24)$; en las mujeres en las escalas de ansiedad y psicoticismo del SCL-90 ( $t=14,31$ y 9,22 respectivamente) y de ansiedad y estrés del DASS-21 $(t=9,89$ y 14,85 respectivamente).

\section{Discusión}

Este estudio ha intentado dimensionar la presencia de trastornos de la salud mental en una muestra representativa de una universidad regional chilena. La caracterización obtenida muestra una población estudiantil aparentemente sana pues las puntuaciones grupales son similares a las puntuaciones indicativas de no sintomatología en los diferentes instrumentos ${ }^{11,20,21}$. Sin embargo, hay algunos resultados inquietantes.

Se confirma que ser mujer es un factor de vulnerabilidad en prácticamente todos los ámbitos evaluados, coincidiendo con lo encontrado en estudios en población universitaria ${ }^{11,16}$ y población general chilena ${ }^{31,32}$. Condicionantes socioculturales y biológicas ${ }^{33}$ permitirían que las estudiantes perciban como amenazas muchos de los eventos vitales a los que se ven enfrentadas, incidiendo negativamente en su salud mental.

En general, la presencia de nivel socioeconómico bajo y características propias de la región como la ruralidad y la pertenencia a la etnia mapuche están asociadas a sintomatología. Estas variables, junto al alto consumo de alcohol y el déficit en conductas de autocuidado y recreación, condicionan negativamente la salud mental de los estudiantes. 
Tabla 3. Estadísticos descriptivos según agrupación por sintomatología y diferencias de grupos para hombres y mujeres en las escalas específicas del SCL-90-R, el DASS-21 y las escalas de Beck

\begin{tabular}{|c|c|c|c|c|c|c|c|c|c|c|}
\hline \multirow[t]{2}{*}{ Escalas } & \multicolumn{5}{|c|}{ Hombres } & \multicolumn{5}{|c|}{ Mujeres } \\
\hline & \multicolumn{2}{|c|}{$\begin{array}{c}\text { Sin } \\
\text { sintomatología } \\
n=192\end{array}$} & \multicolumn{2}{|c|}{$\begin{array}{c}\text { Con } \\
\text { sintomatología } \\
n=25\end{array}$} & $t^{*}$ & \multicolumn{2}{|c|}{$\begin{array}{c}\text { Sin } \\
\text { sintomatología } \\
n=215\end{array}$} & \multicolumn{2}{|c|}{$\begin{array}{c}\text { Con } \\
\text { sintomatología } \\
n=52\end{array}$} & $t^{*}$ \\
\hline \multicolumn{11}{|l|}{ SCL-90 } \\
\hline Somatización & 40,05 & 10,34 & 54,16 & 10,90 & $-6,35$ & 45,15 & 10,17 & 57,73 & 9,77 & $-8,06$ \\
\hline Obsesiones & 46,38 & 11,84 & 66,12 & 14,06 & $-7,67$ & 47,66 & 11,17 & 70,81 & 26,74 & $-6,12$ \\
\hline Sens. Interp. & 44,66 & 10,01 & 59,80 & 13,54 & $-6,80$ & 45,96 & 13,37 & 68,79 & 28,72 & $-5,59$ \\
\hline Depresión & 41,61 & 10,39 & 61,00 & 6,96 & $-12,25$ & 46,23 & 14,23 & 64,75 & 15,49 & $-8,28$ \\
\hline Ansiedad & 38,67 & 10,79 & 56,88 & 8,48 & $-8,11$ & 40,43 & 10,25 & 60,06 & 8,51 & $-14,31$ \\
\hline Hostilidad & 45,25 & 9,96 & 61,80 & 9,52 & $-7,85$ & 45,80 & 10,59 & 65,04 & 21,18 & $-6,35$ \\
\hline Ans. Fóbica & 46,03 & 5,77 & 55,28 & 8,01 & $-5,59$ & 47,12 & 6,94 & 57,58 & 10,83 & $-6,64$ \\
\hline Ideac. Paran. & 45,91 & 8,13 & 61,28 & 7,46 & $-8,97$ & 45,99 & 8,43 & 56,75 & 8,72 & $-8,21$ \\
\hline Psicoticismo & 43,80 & 8,69 & 59,32 & 12,08 & $-6,22$ & 43,81 & 8,08 & 55,29 & 7,94 & $-9,22$ \\
\hline \multicolumn{11}{|l|}{ DASS-21 } \\
\hline Depresión & 2,90 & 2,77 & 8,08 & 4,00 & $-6,28$ & 3,30 & 3,21 & 8,27 & 5,02 & $-6,76$ \\
\hline Ansiedad & 2,14 & 2,02 & 6,72 & 3,37 & $-6,64$ & 2,44 & 2,32 & 7,90 & 3,82 & $-9,89$ \\
\hline Estrés & 3,98 & 2,87 & 10,08 & 3,14 & $-9,24$ & 4,25 & 3,15 & 11,65 & 3,38 & $-14,85$ \\
\hline BAI & 6,69 & 4,50 & 20,60 & 4,37 & $-14,58$ & 8,16 & 5,09 & 23,04 & 6,78 & $-14,84$ \\
\hline BDI-II & 11,25 & 7,36 & 27,00 & 5,91 & $-10,27$ & 13,46 & 7,62 & 29,06 & 8,06 & $-13,10$ \\
\hline
\end{tabular}

Nota. Sin sintomatología $=$ BAI-BDI $<$ PC; con sintomatología $=$ BAI-BDI $>$ PC. Se destacan en negritas los incrementos más significativos del grupo sintomático en las escalas específicas del SCL-90-R y el DASS-21 según su valor t. * $p<0,001$ para la prueba t de Student en todas las escalas consideradas.

La sintomatología depresiva y ansiosa es claramente la más frecuente (entre $30 \%$ y $20 \%$ de la muestra total) acentuándose en los estudiantes de primer año (aproximadamente $60 \%$ con sintomatología ansiosa-depresiva). Este fenómeno se relaciona con que muchos universitarios se encuentran en la adolescencia tardía, etapa en que tienden a emerger problemas de salud mental ${ }^{3}$. Otro factor asociado es el ingreso a la Universidad, etapa de transición caracterizada por cambios, desafíos y ajustes ${ }^{34}$ que afectan a los estudiantes de primer año y que incluye adaptaciones a entornos nuevos y estilos de vida más autónomos vinculados a la salida del hogar paterno, el desarrollo de nuevas relaciones interpersonales y la formación de hábitos de estudios diferentes a los de la Enseñanza Media.

En la muestra de estudio la situación descrita se asocia, además, a altos niveles de estrés medido a través de una escala específica del DASS- $21^{21}$. El estrés afecta la calidad de vida de los universitarios, se relaciona con los altos grados de exigencia, competitividad y expectativas que caracterizan al mundo universitario y aumenta el riesgo de desarrollar cuadros psicopatológicos ${ }^{6,7}$. Tal como aparece en este estudio, es posible que ocasione, más allá de depresión y ansiedad, efectos diferenciados según sexo observándose, en los hombres, mayor ideación paranoide y, en las mujeres, mayor psicoticismo.

En suma, la caracterización obtenida en esta investigación corrobora resultados previos sobre prevalencia de trastornos de salud mental en estudiantes universitarios chilenos ${ }^{11,16}$, altos niveles de consumo de alcohol y drogas ${ }^{15}$, presencia significativa de ansiedad y depresión ${ }^{11,14}$, princi- 
palmente en mujeres ${ }^{16} \mathrm{y}$ en estudiantes de primer año. Además, agrega información relevante sobre la escasa participación de los jóvenes en actividades de recreación, la presencia de altos niveles de estrés, y la vulnerabilidad asociada a bajo nivel socioeconómico y a variables propias del contexto regional (ruralidad, etnia mapuche) que han sido identificadas como factores de riesgo ${ }^{32}$.

Esta información, relevante para actual el sistema universitario chileno, ha permitido diseñar y desarrollar en la Universidad Austral de Chile actividades de prevención de trastornos y promoción de la salud mental de los estudiantes cuyo objetivo ha sido apoyarlos para afrontar satisfactoriamente las tareas académicas y sociales propias del ámbito universitario, favoreciendo un desarrollo personal integral y promoviendo estilos de vida saludables. Específicamente, se han creado programas de intervención dirigidos a los estudiantes de primer año, ya que son ellos quienes se encuentran en el periodo más vulnerable.

\section{Referencias}

1. Goic A. Proliferación de escuelas de medicina en Latinoamérica: causas y consecuencias. Rev Med Chile 2002; 130: 917-24.

2. Meneses F, Rolando R, Valenzuela M, Vega M. Ingreso a la Educación Superior: La experiencia de la cohorte de egreso 2005. División de Educación Superior, Ministerio de Educación de Chile. Disponible en http://www.divesup.cl/images/archivos/Publicaciones/ingreso $\% 20 a \% 20$ la\%20educacin\%20superior. [Consultado el 22 de Junio de 2010].

3. Harrington R. Affective disorders. En: Rutter M y Taylor E. Child and Adolescent Psychiatry. Oxford, England: Blackwell Publishing Science; 2002: 463-85.

4. Florenzano R, Valdés M. El adolescente y sus conductas de riesgo. Santiago, Chile: Ediciones UC; 2005.

5. Obiols J. Marcadores de riesgo para trastornos del espectro esquizofrénico en la adolescencia. En J. Buendía (Ed.). Psicopatología en Niños y Adolescentes: Desarrollos Actuales. Madrid, España: Pirámide; 1996: 247-64.

6. Aracena M, Barrientos P, Rehbein L. Prevalencia de trastornos emocionales en estudiantes de la universidad de la frontera. Revista Frontera 1992; 11: 19-27.

7. Caparrós B, Viñas F. Afrontamiento del periodo de exámenes y sintomatología somática autoinformada en un grupo de estudiantes universitarios. Revista Electrónica de Psicología; 2000: 4. Disponible en http://www.psi- quiatria.com/revistas/index.php/psicologiacom/article/ view/653/629/. [Consultado el 15 de Abril de 2008].

8. Reeve J. Motivación y emoción. Madrid, España: Mc Graw-Hill; 1994.

9. Simón M. Manual de psicología de la salud. Fundamentos, metodología y aplicaciones. Madrid, España: Biblioteca Nueva; 1999.

10. Goldberg D, Hillier V. A scaled version of the general health questionnaire. Psychological Medicine 1979; 9, 139-45.

11. Cova F, Alvial W, Aro M, Bonifetti A, Hernández M, Rodríguez C. Problemas de salud mental en estudiantes de la Universidad de Concepción. Ter Psicol 2007; 25: 105-12.

12. Beck A, Steer R, Brown G. BDI-II. Beck Depression Inventory. Manual. Second Edition. San Antonio, United States: The Psychological Corporation; 1996.

13. Beck A, Steer R. Beck Anxiety Inventory Manual. San Antonio, United States: The Psychological Corporation; 1990.

14. Rioseco P, Valdivia S, Vicente B, Vielma M, Jerez M. Nivel de salud mental en los estudiantes de la Universidad de Concepción. Revista de Psiquiatría 1996; 11: 158-65.

15. Centro de Documentación e Información del Consejo Nacional para el Control de Estupefacientes. Construyendo contextos preventivos en educación superior. Orientaciones para la formulación de políticas de prevención del consumo de drogas y alcohol. Santiago, Chile: Andros; 2007.

16. Fritsch R, Escanilla X, Goldsack V, Grinberg A, Navarrete A, Pérez A, et al. Diferencias de género en el malestar psíquico de estudiantes universitarios. Revista de Psiquiatría Clínica 2006; 43: 22-30.

17. Micin S, Bagladi V. Salud mental en estudiantes universitarios: incidencia de psicopatología y antecedentes de conducta suicida en población que acude a un servicio de salud estudiantil. Ter Psicol 2011; 29: 53-64.

18. ADIMARK. El Nivel Socio Económico ESOMAR, Manual de Aplicación. ADIMARK 2000. Disponible en http://www.microweb.cl/idm/documentos/ESOMAR. pdf. [Consultado el 15 Junio de 2010]

19. Derogatis, L. R. (1994). SCL-90-R. Administration, Scoring and Procedures Manual (Third Edition). Minneapolis: National Computer Systems.

20. Gempp R, Avendaño C. Datos normativos y propiedades psicométricas del SCL-90-R en estudiantes universitarios chilenos. Ter Psicol 2008; 26: 39-58.

21. Antúnez Z, Vinet E. Escalas de Depresión, Ansiedad y Estrés (DASS-21): Validación de la Versión Abreviada en Estudiantes Universitarios Chilenos. Ter Psicol 2012; 30: (en prensa). 
22. Antony M, Bieling P, Cox B, Enns M, Swinson R. Psychometric properties of the 42 -item and 21-item versions of the Depression Anxiety Stress Scales in clinical groups and a community sample. Psychological Assessment 1998; 10: 176-81.

23. Clara I, Cox B, Enns M. Confirmatory factor analysis of the Depression Anxiety Stress Scales in depressed and anxious patients. Journal of Psychopathology and Behavioral Assessment 2001; 23: 61-7.

24. Apóstolo J, Mendes A, Azeredo Z. Adaptación para la lengua portuguesa de la depression, anxiety and stress scale (DASS). Revista Latino-Americana de Enfermagem 2006; 14: 863-71.

25. Daza P, Novy D, Stanley M, Averill P. The Depression Anxiety Stress Scale-21: Spanish translation and validation with a Hispanic sample. Journal of Psychopathology and Behavioral Assessment 2002; 24: 195-205.

26. Bados A, Solanas A, Andrés R. Psychometric properties of the Spanish version of Depression, Anxiety and Stress Scales (DASS). Psicothema 2005; 17: 679-83.

27. Cova F, Melipillán R, Valdivia M, Bravo E, Valenzuela B. Sintomatología depresiva y ansiosa en estudiantes de enseñanza media. Revista Chilena de Pediatría 2007; 78:
151-9.

28. Beck A, Epstein N, Brown G, Steer R. An inventory for measuring clinical anxiety: Psychometric properties. Journal of Consulting and Clinical Psychology 1988; 56 : 893-7.

29. Sanz J, Navarro M, Vázquez C. Adaptación española del Inventario para la Depresión de Beck-II (BDI-II): Propiedades psicométricas en estudiantes universitarios. Análisis y Modificación de Conducta 2003; 29: 239-88.

30. Cova F, Melipillán R, Rincón P, Valdivia M. Propiedades psicométricas de Inventario de Depresión de Beck-II en adolescentes chilenos. Ter Psicol 2008; 26: 59-69.

31. Vicente P, Rioseco P, Saldivia S, Kohn R, Torres S. Estudio chileno de prevalencia de patología psiquiátrica (DSM-III-R/CIDI) (ECPP). Rev Med Chile 2002; 130: 527-36.

32. Vicente P, Kohn R, Saldivia S, Rioseco P. Carga del enfermar psíquico, barreras y brechas en la atención de Salud Mental en Chile. Rev Med Chile 2007; 135: 1591-9.

33. Fliman M. Un diagnóstico de la situación de la salud mental en Chile. Rev Med Chile 1972; 100 (3): 343-8.

34. Gimeno J. La transición a la educación secundaria. Madrid, España: Morata; 1997. 